\title{
Should ureteroscopy be performed for patients after ureteral reconstruction with autologous onlay flap/graft?
}

\author{
Jie Wang ${ }^{1 \#}$, Shubo Fan ${ }^{1 \#}$, Hua Guan ${ }^{1 \#}$, Shengwei Xiong ${ }^{1}$, Dengxiang Zhang ${ }^{2}$, Bingwei Huang ${ }^{3}$, \\ Xiang Wang ${ }^{1}$, Hongjian Zhu ${ }^{2}$, Zhihua Li $^{1}$, Gengyan Xiong ${ }^{1}$, Zhongyuan Zhang ${ }^{1}$, Kunlin Yang ${ }^{1}$, \\ Xuesong $\mathrm{Li}^{1}$, Liqun Zhou ${ }^{1}$ \\ ${ }^{1}$ Department of Urology, Peking University First Hospital, Institute of Urology, Peking University, National Urological Cancer Center, Beijing, \\ China; ${ }^{2}$ Department of Urology, Beijing Jiangong Hospital, Beijing, China; ${ }^{3}$ Department of Urology, Emergency General Hospital, Beijing, China \\ Contributions: (I) Conception and design: J Wang, S Fan; (II) Administrative support: X Li, L Zhou; (III) Provision of study materials or patients: K \\ Yang, X Li; (IV) Collection and assembly of data: H Guan, Z Li, D Zhang, B Huang, H Zhu; (V) Data analysis and interpretation: S Xiong, X Wang, \\ G Xiong, Z Zhang; (VI) Manuscript writing: All authors; (VII) Final approval of manuscript: All authors. \\ \#These authors contributed equally to this work. \\ Correspondence to: Kunlin Yang; Xuesong Li. Department of Urology, Peking University First Hospital, Institute of Urology, Peking University, National \\ Urological Cancer Center, No. 8 Xishiku St, Xicheng District, Beijing 100034, China. Email: yangkunlin12345@163.com; pineneedle@sina.com.
}

Background: To analyze the safety and clinical significance of performing ureteroscopy after ureteral reconstruction with autologous onlay/graft. To describe the ureteroscopic appearances of the appendiceal onlay flap and lingual mucosa graft.

Methods: Beginning in August 2018, we conducted a prospective cohort study of autologous onlay/graft techniques to repair ureteral strictures. The perioperative data of 42 patients who had undergone surgery more than 6 months prior were collected prospectively. During the postoperative follow-up, ureteroscopy was performed in 27 patients (64.3\%) after surgery (group A), and ureteroscopy was not performed in the other 15 patients (35.7\%) (group B). We carried out a comparative study of these two groups of patients. Analyses were conducted mainly on complications related to ureteroscopy and the success rate of ureteral reconstruction surgery.

Results: There were no significant differences in patient demographic data or the length of ureteral reconstruction between the two groups $(\mathrm{P}>0.05)$. For the ureteroscopy group, the median time from repair surgery to ureteroscopy was 3 (range, 2-7) months, there was no poor healing of the anastomosis, and the ureteral lumen of all patients was unobstructed. Some expected observations can be found in the ureteral lumen, such as mucosa edema, stones, follicles and granulation tissue. Among the 27 patients, one patient (3.7\%) developed bleeding intraoperatively and 7 patients $(25.9 \%)$ were found to have low-grade (ClavienDindo I and II) postoperative complications, including 5 cases of fever and 2 cases of bleeding. The mean

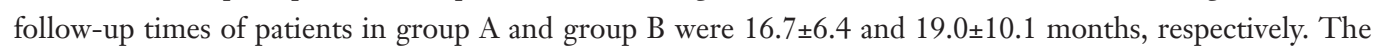
objective success (imaging showed hydronephrosis ease) rate of the two groups was $100 \%$. The subjective success (symptom relief) rates of group A and group B were $96.3 \%$ and $100 \%$, respectively.

Conclusions: Patients after autologous onlay flap/graft ureteroplasty do not need to undergo routine ureteroscopy unless there is aggravation of hydronephrosis or other indications for ureteroscopy, such as stones.

Keywords: Ureteral reconstruction; autologous onlay; ureteroscopy; appendix; lingual mucosa

Submitted Jul 02, 2021. Accepted for publication Aug 26, 2021.

doi: $10.21037 /$ tau-21-583

View this article at: https://dx.doi.org/10.21037/tau-21-583 


\section{Introduction}

Ureteral stenosis can be managed by reconstructive surgery. However, many individual factors, such as the length, sites and severity of ureteral strictures, can make repair surgery extremely challenging $(1,2)$. In recent years, with the improvement in surgical techniques and the innovation of upper urinary tract reconstruction concepts, the onlay technique has been gradually used to repair complicated ureteral strictures. The most commonly used autologous tissues in clinical practice include appendiceal onlay flaps (AOFs) and oral mucosa grafts (3-9). The concepts of these two methods are identical, but the practical implementation is slightly different. At present, there have been few cases reported worldwide. Therefore, there is no mature experience in the perioperative management of AOF ureteroplasty and oral mucosa graft ureteroplasty, especially in the postoperative examination. Whether ureteroscopy should be performed after surgery is a question worth discussing. Although ureteroscopy can visually observe the healing of the onlay flaps or grafts and the patency of the lumen at anastomosis, it is an invasive examination with some complications. Duty et al. suggested that if there was concern about persistent obstruction after AOF ureteroplasty, ureteroscopy should be performed (4). Zhao et al. reported that they performed ureteroscopy after the buccal mucosa graft was anastomosed to the ureter intraoperatively to confirm a patent and watertight anastomosis (10).

However, to date, there are almost no studies about the healing of onlay flaps or grafts and whether the lumen at the anastomosis is narrowed, as observed through ureteroscopy postoperatively. The purpose of the present study was to describe the ureteroscopic appearances of AOF and lingual mucosa graft (LMG). We also analyzed the safety and clinical significance of performing ureteroscopy after ureteral reconstruction with the onlay technique.

We present the following article in accordance with the STROBE reporting checklist (available at https://dx.doi. org/10.21037/tau-21-583).

\section{Methods}

After the approval of the hospital ethics committee, we conducted a prospective cohort study of the onlay technique to repair ureteral strictures beginning in August 2018. To observe the healing of the onlay flap/graft and provide experience with this rare procedure, we conducted an observational study on routine ureteroscopy after onlay flap/graft ureteroplasty. This study approved by the Ethics Committee of Peking University First Hospital (ethical approval number: 2019SR134).

\section{AOF ureteroplasty}

This procedure is usually used to repair proximal and middle strictures of the right ureter. The appendix was separated from the cecum with its mesoappendix preserved. The free end of the appendix was transected and detubularized along its antimesenteric border. The severity of ureteral stenosis can be divided into two different types and requires different strategies with AOF. If the ureteral stricture could be incised longitudinally along the anterior wall and the posterior wall of the ureteral stricture was integral, the AOF was anastomosed to the anterior wall defect of the ureteral stricture. Otherwise, the ureteral lumen was obliterative. We resected the ureteral obliterative segment and then anastomosed the normal ureteral tissue with the posterior wall to create a new ureteral plate, and then the AOF was anastomosed to the anterior wall defect. This is the posteriorly augmented anastomotic technique $(6,7)$. In our study, 13 patients underwent AOF ureteroplasty with 6 laparoscopic procedures and 7 robotic procedures. Nine patients underwent the posteriorly augmented anastomotic technique.

\section{LMG ureteroplasty}

In our institution, this procedure is usually used to repair proximal and middle strictures of the left ureter and right ureteral stenosis, which is not suitable for AOF ureteroplasty. The sites of the harvesting grafts were usually marked on the ventral surface of the tongue. The grafts were tailored to match the size of the ureteral stricture. First, diluted epinephrine $(1: 100,000)$ was injected into the submucosa of the tongue, which can facilitate graft harvesting and minimize bleeding. Then, the submucosal muscle and adipose tissue of the graft were removed to create an appropriate thickness and size graft. Finally, the donor site was sutured with continuous running with 3-0 Vicryl to avoid bleeding (5). The prepared LMG was placed in the abdominal cavity and anastomosed to the ureteral stricture. Similarly, the posteriorly augmented anastomotic technique can also be used with LMG ureteroplasty (9). We included 29 patients who had undergone LMG ureteroplasty, with 14 laparoscopic procedures and 15 robotic procedures. 
Fourteen patients underwent the posteriorly augmented anastomotic technique simultaneously.

\section{Postoperative ureteroscopy}

Under general anesthesia, patients were placed in a lithotomy position. An F6/7.5 rigid ureteroscope (Richard Wolf, Knittlington, Germany) was inserted through the urethra and into the bladder, and the old double-J stent was removed. Then, a smooth guide wire was inserted into the ureter, the full length of the ureter was observed through ureteroscopy, and the site of onlay anastomosis was checked carefully. Finally, a new double-J stent was placed. Antibiotics were given for 3-5 days postoperatively. We performed ureteroscopy for each patient 2-3 months after surgery to observe the healing morphology of the onlay graft/flap, excluding patients who refused to be examined when we started ureteral reconstruction with the onlay technique. Gradually, we have accumulated more mature experience in anastomosis techniques and mastered the healing of the onlay graft/flap. However, some complications related to ureteroscopy also occurred. Therefore, we stopped performing ureteroscopy routinely for patients in July 2020, unless there is aggravation of hydronephrosis or other indications for ureteroscopy, such as stones.

\section{Follow-up after ureteral reconstruction}

Patients with a nephrostomy tube underwent IUE after removing the double-J stent (7). Patients without a nephrostomy tube underwent cine MRU after removing the double-J stent. Then, patients underwent ultrasound three months after surgery. Finally, patients paid attention to their symptoms, such as flank pain and fever, and a repeat ultrasound was performed six months later and then annually. The effect of surgery was evaluated by two criteria. Subjective success was defined as the resolution of flank pain without a double-J stent and nephrostomy. Objective success was defined as the absence of ureteral obstruction on imaging examinations. The success rate was evaluated after at least a follow-up time of 6 months.

\section{Statistical analysis}

Data were analyzed using SPSS software version 22.0 (IBM Corporation, Armonk, NY, USA). Measurement data are expressed as the mean \pm standard deviation, and enumeration data are expressed as numbers (percentages). Comparisons between groups were performed using the Mann-Whitney $\mathrm{U}$ test and the chi-square test. $\mathrm{P}$ values $<0.05$ were considered statistically significant.

\section{Ethical statement}

The study was conducted in accordance with the Declaration of Helsinki (as revised in 2013). The study was approved by Ethics Committee of Peking University First Hospital (NO.: 2019SR134) and individual consent for this retrospective analysis was waived.

\section{Results}

To date, the perioperative data of 14 patients who underwent AOF ureteroplasty and 33 patients who underwent LMG ureteroplasty have been collected prospectively. In this study, 42 patients who had undergone ureteral reconstruction surgery for more than 6 months were recruited. The demographics and characteristics of all patients are presented in Table 1. The median preoperative serum creatinine and estimated glomerular filtration rate (eGFR) of all patients were $80.3(11.4-212.0) \mu \mathrm{mol} / \mathrm{L}$ and $94.8(46.8-134.2) \mathrm{mL} / \mathrm{min} / 1.73 \mathrm{~m}^{2}$, respectively. Sixteen patients had clear urinary tract infection (UTI) before operation. Nephrostomy was performed in 22 patients and double-J ureteral stent was indwelt in 10 patients, before their ureteral reconstruction surgery. In addition, there were 15 patients had previous management for ureteral strictures, including pyeloplasty, ureteroureterostomy, endoureterotomy and balloon dilatation. In present study, all procedures were performed successfully. Thirteen of $42(31.0 \%)$ patients underwent AOF ureteroplasty, and there were no complications at the harvest site and other intestinal complications after surgery. Twenty-nine of $42(69.0 \%)$ patients underwent LMG ureteroplasty, and 4 patients $(13.8 \%)$ had tongue numbness, which were gradually improved within one week after surgery. During the postoperative follow-up, noninvasive examinations such as ultrasound, computed tomography (CT), imaging urodynamics examination (IUE), or cine magnetic resonance urography (cine MRU) were implemented in all patients. In addition, ureteroscopy was performed in 27 patients $(64.3 \%) 2-3$ months after surgery, who were classified as group A, and 15 patients (35.7\%) did not undergo ureteroscopy, who were classified as group B. There were no significant differences in patient 
Table 1 The demographic and characteristics for patients of two groups

\begin{tabular}{|c|c|c|c|c|}
\hline Parameter & Overall & Ureteroscopy & No ureteroscopy & $\mathrm{P}$ \\
\hline Sex, n (\%) & & & & 0.921 \\
\hline Male & $30(71.4)$ & $19(70.4)$ & $11(73.3)$ & \\
\hline Female & $12(28.6)$ & $8(29.6)$ & $4(26.7)$ & \\
\hline $\mathrm{BMI}\left(\mathrm{kg} / \mathrm{m}^{2}\right)$, mean $\pm \mathrm{SD}$ & $23.7 \pm 3.7$ & $24.3 \pm 3.5$ & $22.6 \pm 4.0$ & 0.156 \\
\hline \multicolumn{5}{|l|}{ Onlay tissues, n (\%) } \\
\hline Appendiceal onlay flap & $13(31.0)$ & $9(33.3)$ & $4(26.7)$ & \\
\hline Lingual mucosa graft & $29(69.0)$ & $18(66.7)$ & $11(73.3)$ & \\
\hline Robot & $22(52.4)$ & $15(55.6)$ & $7(46.7)$ & \\
\hline Laparoscopy & $20(47.6)$ & $12(44.4)$ & 8 (53.3) & \\
\hline
\end{tabular}

demographic data or the length of ureteral reconstruction between the two groups $(\mathrm{P}>0.05)$ (Table 1). For group A, the median time from repair surgery to ureteroscopy was 3 (range, 2-7) months, and among them, there were 11 patients with intervals of 2 and 3 months. Due to the impact of COVID-19, ureteroscopy was performed in two patients 6 and 7 months after surgery. For all ureteroscopy procedures, no poor healing of the anastomosis was observed, and each ureteral lumen was watertight. The ureteral lumen of all patients was unobstructed. For patients 2 months after surgery, edema was still present in the ureteral mucosa, especially around the anastomosis. For patients more than 3 months after repair operations, the edema of anastomosis improved slightly. However, this phenomenon was not universal. There was a patient with solitary kidney and ureteral stenosis who underwent LMG ureteroplasty in our hospital. The double-J stent was removed without ureteroscopy 2 months after surgery. Four months after surgery, the patient developed flank pain, and ingravescent hydronephrosis was found by ultrasound. To clarify the cause of the hydronephrosis and relieve it, we performed ureteroscopy 5 months after surgery. The results showed that there was still edema in the ureteral mucosa but no obvious stenosis, and a new double-J stent was indwelled. In addition, most patients had flocs in the ureteral lumen during ureteroscopy, 3 patients were found to have ureteral stones, and two patients were found to have follicles or granulation tissue at the anastomosis (Figure 1).

In group A, some intraoperative and early postoperative complications related to ureteroscopy were recorded. One patient of 27 (3.7\%) developed bleeding during the ureteroscopy procedure, but visibility allowed the completion of ureteroscopy. Some low-grade (ClavienDindo I and II) postoperative complications were observed within one week after ureteroscopy. Five of $27(18.5 \%)$ patients developed fever $\left(\max 39.4{ }^{\circ} \mathrm{C}\right)$ postoperatively, accompanied by elevated white blood cells and procalcitonin, which are reliable indicators of bacterial infection. Two patients' urine cultures showed positive Escherichia coli among the five abovementioned patients. All 5 patients responded well to carbapenem antibiotics. Two of 27 (7.4\%) patients had bleeding from the nephrostomy tube and urinary tube after operations accompanied by low back pain. The bleeding disappeared gradually through ingestion of drinking water and diuresis. The patients in group B did not undergo ureteroscopy and had no ureteroscopy-related complications. The ureteroscopy-related complications of the two groups are summarized in Table 2.

The mean follow-up times of patients with and without ureteroscopy were $16.7 \pm 6.4$ months and $19.0 \pm 10.1$ months, respectively. The follow-up time of each patient was more than 6 months. The objective success rate of the two groups 

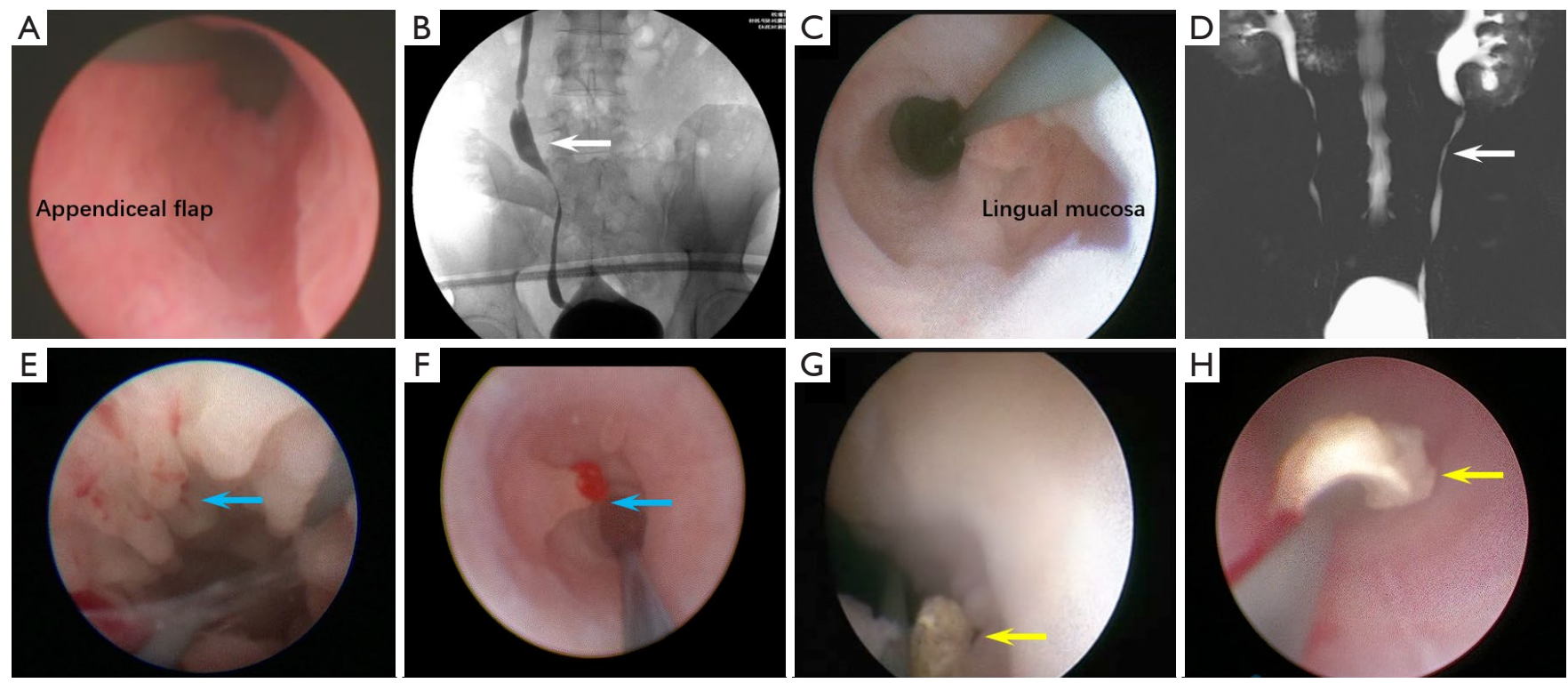

Figure 1 The appearances observed under ureteroscopy after ureteral reconstruction with autologous onlay flap/graft. (A) Mucosal redness and swelling can be seen at the anastomosis of the AOF and ureter. (B) IUE at 3 months after AOF right ureteroplasty showing an unobstructed ureter. AOF can be seen (white arrow). (C) The ureteral lumen is unobstructed at the anastomosis of the LMG and the ureter. (D) Cine MRU at 3 months after LMG left ureteroplasty showing an unobstructed ureter. The LMG can be identified (white arrow). (E) Follicular-like tissue on the ureteral mucosa (blue arrow). (F) Granulation or polyps around the anastomosis (blue arrow). (G) A free round stone in the ureteral lumen (yellow arrow). $(\mathrm{H})$ The fragments of stones that appended to the double-J stent fell off in the ureteral lumen (yellow arrow).

Table 2 The ureteroscopy-related complications and the outcomes of repair surgery of two groups

\begin{tabular}{lcc}
\hline Parameter & Ureteroscopy No ureteroscopy \\
\hline $\begin{array}{l}\text { Ureteroscopy-related } \\
\text { complications, } \mathrm{n}(\%)\end{array}$ & & \\
Intraoperative bleeding & $1(3.7)$ & 0 \\
Postoperative fever & $5(18.5)$ & 0 \\
Postoperative bleeding & $2(7.4)$ & \\
Outcomes of repair surgery (\%) & & 100 \\
Subjective success rate & 96.3 & 100 \\
Objective success rate & 100 & \\
\hline
\end{tabular}

was $100 \%$. The subjective success rate of group A was $96.3 \%(26 / 27)$. One patient who was 6 months after surgery had intermittent low back pain that could be relieved by oral antibiotics. The subjective success rate of group B was $100 \%$. The outcomes of repair surgery in the two groups are summarized in Table 2. In general, ureteroscopy provides us with the ability to observe the healing of AOFs and LMGs. However, according to the current follow-up, it did not affect the success rate of the ureteral reconstruction surgery.

\section{Discussion}

Surgical management of proximal and middle ureteral strictures presents a challenge to urologists. Based on the encouraging results achieved using onlay grafts in urethral stricture treatment (11), the same reconstructive principles have been applied to ureteral strictures (12). Currently, ureteroplasty with oral mucosal grafts or AOF has been increasingly utilized to repair long-segment stenosis of the proximal and middle ureters (3-6,8,13-15). Although there was no self-rejection reaction, the oral mucosal grafts and $\mathrm{AOF}$ were new tissues sutured to the ureter. In addition, the oral mucosal graft is a free graft without blood supply, and the AOF is an onlay with blood supply. However, there is a paucity of literature presenting the healing appearance of grafts and flaps under ureteroscopy. In our study, there was no poor healing of the anastomosis in any of the 
27 ureteroscopy procedures, and each ureteral lumen was watertight. Both the AOF and the LMG healed well, which confirmed the feasibility of the onlay technique for ureteral repair. We believe that ureteroscopy for patients 2-3 months after surgery can not only observe whether the anastomosis is stenotic or poorly healed but can also enable the surgeon to obtain feedback that can improve his or her suturing skills, especially in the early stages of performing onlay surgery.

However, ureteroscopy is an invasive examination, especially when using rigid ureteroscopes (16-18). Geavlete et al. reported a single-center experience of complications among 2,735 retrograde semirigid ureteroscopy procedures. The rate of intraoperative complications was $3.6 \%$, including mucosal injury (2.5\%), ureteral perforation $(0.65 \%)$, extraureteral stone migration $(0.18 \%)$, bleeding $(0.1 \%)$, and ureteral avulsions $(0.11 \%)$. The early postoperative complication rate was $10.64 \%$, including fever or sepsis $(1.13 \%)$, persistent hematuria $(2.04 \%)$, renal colic $(2.23 \%)$, migrated double- $\mathrm{J}$ stent $(0.66 \%)$, and transitory vesicoureteral reflux $(4.58 \%$, especially in cases with indwelling double-J stents) (19). de la Rosette et al. assessed the postoperative complication rate for ureteroscopy among 11,885 patients and found that it was $3.5 \%$, and the most frequent complication was fever (1.8\%) (20). In addition, Berardinelli et al. reported a multicenter review of infectious complications after ureteroscopy and noted a $4.4 \%$ risk of postoperative fever and a $0.7 \%$ risk of sepsis in 403 patients from five centers (21). However, some urologists reported that the most common complication in ureteroscopic lithotripsy was mucosal injury (52.6-62\%) (22-24). In our study, the intra- and postoperative complication rates of the ureteroscopy group were $3.7 \%$ (one patient experienced bleeding) and $25.9 \%$ (five patients had fever and two patients experienced bleeding), respectively. According to our experience, intra- and postoperative bleeding is associated with mucosal injury, which is usually minor and self-limited $(18,25)$. Postoperative fever was associated with bacterial colonization in the urinary tract or ureteral stents and the hydrostatic pressure generated by the irrigation fluid during ureteroscopy. Several studies have indicated that preoperative hydronephrosis, pyelonephritis, bacteriuria, preoperative positive urine culture, longer operative time, and indwelling drainage tubes are all associated with postoperative fever $(26,27)$. The patients in our study usually had a history of hydronephrosis and long-term indwelling double-J tubes before ureteral repair surgery, which increased the risk of postureteroscopy fever.
In addition, the risk of long-term complications existed after ureteroscopy, such as ureteral stenosis (25).

In our study, ureteroscopy was an examination method used after autologous onlay flap/graft ureteroplasty. We used the success rate of ureteral repair surgery to indirectly reflect the effectiveness and clinical significance of ureteroscopy. There was no significant difference in the surgical success rate between the ureteroscopy group and the nonureteroscopy group. We believe that ureteroscopy, in addition to obtaining intuitive images of autologous onlay flaps/grafts, can be replaced by other imaging examinations in the assessment of ureteral patency. Of course, diagnostic ureteroscopy is simple in operation and requires a minimum amount of operation time, which is different from therapeutic ureteroscopy, such as lithotripsy. The risk of serious complications is very low. We did not find any cases of failed repair surgery due to ureteroscopy. In addition, compared to the nonureteroscopy group, patients in the ureteroscopy group must undertake the risks of the procedure and general anesthesia, as well as the surgical cost. Therefore, based on the experience of 27 ureteroscopic procedures, we believe that ureteroscopy is not a necessary examination for patients after autologous onlay flap/graft ureteroplasty. However, for patients whose hydronephrosis gradually worsens after removing double-J stents, along with impaired renal function or severe renal colic, in whom a new double-J stent should be indwelled, ureteroscopy should be performed to determine the cause of hydronephrosis, especially for patients with a solitary kidney. In addition, patients with stones can undergo ureteroscopic lithotripsy while observing the autologous onlay flap/graft.

Our study was potentially limited by its small sample size, observational design and lack of randomization between groups, which may result in selection bias. Although the results of the present study must be interpreted given the above limitations, we described the significance and complications of ureteroscopy after ureteral reconstruction with the onlay technique based on specific clinical practice and provided some suggestions for urologists dedicated to upper urinary tract reconstruction.

\section{Conclusions}

Patients after autologous onlay flap/graft ureteroplasty do not need to undergo routine ureteroscopy unless there is aggravation of hydronephrosis or other indications for ureteroscopy, such as stones. 


\section{Acknowledgments}

The authors are grateful for the entire staff of the Department of Urology, Peking University First Hospital. Funding: None.

\section{Footnote}

Reporting Checklist: The authors have completed the STROBE reporting checklist. Available at https://dx.doi. org/10.21037/tau-21-583

Data Sharing Statement: Available at https://dx.doi. org/10.21037/tau-21-583

Peer Review File: Available at https://dx.doi.org/10.21037/ tau-21-583

Conflicts of Interest: All authors have completed the ICMJE uniform disclosure form (available at https://dx.doi. org/10.21037/tau-21-583). XL and LZ serve as unpaid editorial board members. The other authors have no conflicts of interest to declare.

Ethical Statement: The authors are accountable for all aspects of the work in ensuring that questions related to the accuracy or integrity of any part of the work are appropriately investigated and resolved. The study was conducted in accordance with the Declaration of Helsinki (as revised in 2013). The study was approved by Ethics Committee of Peking University First Hospital (NO.: 2019SR134) and individual consent for this retrospective analysis was waived.

Open Access Statement: This is an Open Access article distributed in accordance with the Creative Commons Attribution-NonCommercial-NoDerivs 4.0 International License (CC BY-NC-ND 4.0), which permits the noncommercial replication and distribution of the article with the strict proviso that no changes or edits are made and the original work is properly cited (including links to both the formal publication through the relevant DOI and the license). See: https://creativecommons.org/licenses/by-nc-nd/4.0/.

\section{References}

1. Engel O, Rink M, Fisch M. Management of iatrogenic ureteral injury and techniques for ureteral reconstruction.
Curr Opin Urol 2015;25:331-5.

2. Xiong SW, Yang KL, Ding GP, et al. Advances in surgical repair of ureteral injury. Beijing Da Xue Xue Bao Yi Xue Ban 2019;51:783-9.

3. Kroepfl D, Loewen H, Klevecka V, et al. Treatment of long ureteric strictures with buccal mucosal grafts. BJU Int 2010;105:1452-5.

4. Duty BD, Kreshover JE, Richstone L, et al. Review of appendiceal onlay flap in the management of complex ureteric strictures in six patients. BJU Int 2015;115:282-7.

5. Li B, Xu Y, Hai B, et al. Laparoscopic onlay lingual mucosal graft ureteroplasty for proximal ureteral stricture: initial experience and 9-month follow-up. Int Urol Nephrol 2016;48:1275-9.

6. Zhao LC, Weinberg AC, Lee Z, et al. Robotic Ureteral Reconstruction Using Buccal Mucosa Grafts: A Multiinstitutional Experience. Eur Urol 2018;73:419-26.

7. Wang J, Xiong S, Fan S, et al. Appendiceal Onlay Flap Ureteroplasty for the Treatment of Complex Ureteral Strictures: Initial Experience of Nine Patients. J Endourol 2020;34:874-81.

8. Jun MS, Stair S, Xu A, et al. A Multi-Institutional Experience With Robotic Appendiceal Ureteroplasty. Urology 2020;145:287-91.

9. Fan S, Yin L, Yang K, et al. Posteriorly Augmented Anastomotic Ureteroplasty with Lingual Mucosal Onlay Grafts for Long Proximal Ureteral Strictures: 10 Cases of Experience. J Endourol 2021;35:192-9.

10. Zhao LC, Yamaguchi Y, Bryk DJ, et al. Robot-Assisted Ureteral Reconstruction Using Buccal Mucosa. Urology 2015;86:634-8.

11. Mangera A, Patterson JM, Chapple CR. A systematic review of graft augmentation urethroplasty techniques for the treatment of anterior urethral strictures. Eur Urol 2011;59:797-814.

12. Reggio E, Richstone L, Okeke Z, et al. Laparoscopic ureteroplasty using on-lay appendix graft. Urology 2009;73:928.e7-10.

13. Lee Z, Keehn AY, Sterling ME, et al. A Review of Buccal Mucosa Graft Ureteroplasty. Curr Urol Rep 2018;19:23.

14. Lee M, Lee Z, Koster H, et al. Intermediate-term outcomes after robotic ureteral reconstruction for longsegment ( $\geq 4$ centimeters) strictures in the proximal ureter: A multi-institutional experience. Investig Clin Urol 2021;62:65-71.

15. Cheng S, Fan S, Wang J, et al. Laparoscopic and robotic ureteroplasty using onlay flap or graft for the management of long proximal or middle ureteral strictures: our 
experience and strategy. Int Urol Nephrol 2021;53:479-88.

16. Francesca F, Scattoni V, Nava L, et al. Failures and complications of transurethral ureteroscopy in 297 cases: conventional rigid instruments vs. small caliber semirigid ureteroscopes. Eur Urol 1995;28:112-5.

17. Schuster TG, Hollenbeck BK, Faerber GJ, et al. Complications of ureteroscopy: analysis of predictive factors. J Urol 2001;166:538-40.

18. Johnson DB, Pearle MS. Complications of ureteroscopy. Urol Clin North Am 2004;31:157-71.

19. Geavlete P, Georgescu D, Niţă G, et al. Complications of 2735 retrograde semirigid ureteroscopy procedures: a single-center experience. J Endourol 2006;20:179-85.

20. de la Rosette J, Denstedt J, Geavlete P, et al. The clinical research office of the endourological society ureteroscopy global study: indications, complications, and outcomes in 11,885 patients. J Endourol 2014;28:131-9.

21. Berardinelli F, De Francesco P, Marchioni M, et al. Infective complications after retrograde intrarenal surgery: a new standardized classification system. Int Urol Nephrol 2016;48:1757-62.

22. Tanriverdi O, Silay MS, Kadihasanoglu M, et al. Revisiting the predictive factors for intra-operative complications of rigid ureteroscopy: a 15 -year experience. Urol J 2012;9:457-64.

23. Fuganti PE, Pires S, Branco R, et al. Predictive factors for intraoperative complications in semirigid ureteroscopy: analysis of 1235 ballistic ureterolithotripsies. Urology 2008;72:770-4.

24. Ather MH, Paryani J, Memon A, et al. A 10-year experience of managing ureteric calculi: changing trends towards endourological intervention--is there a role for open surgery? BJU Int 2001;88:173-7.

25. D'Addessi A, Bassi P. Ureterorenoscopy: avoiding and managing the complications. Urol Int 2011;87:251-9.

26. Moses RA, Ghali FM, Pais VM Jr, et al. Unplanned Hospital Return for Infection following UreteroscopyCan We Identify Modifiable Risk Factors? J Urol 2016;195:931-6.

27. Wollin DA, Joyce AD, Gupta M, et al. Antibiotic use and the prevention and management of infectious complications in stone disease. World J Urol 2017;35:1369-79.
Cite this article as: Wang J, Fan S, Guan H, Xiong S, Zhang D, Huang B, Wang X, Zhu H, Li Z, Xiong G, Zhang Z, Yang K, Li X, Zhou L. Should ureteroscopy be performed for patients after ureteral reconstruction with autologous onlay flap/graft? Transl Androl Urol 2021;10(10):3737-3744. doi: $10.21037 /$ tau-21-583 